Ann. Biol. anim. Bioch. Biophys., I970, 10 (2), 323-326.

\title{
UTILISATION DIGESTIVE DE L'AMIDON DU MAÏS CHEZ LE RUMINANT
}

\author{
P. THIVEND et M. JOURNET \\ Station de Recherches sur l'Élevage des Ruminants, \\ Centre de Recherches de Clermont-Ferrand, 63 -Saint-Genès-Champanelle \\ Institut national de la Recherche agronomique
}

La digestion de l'amidon du maïs dans l'intestin du Ruminant a fait l'objet d'un petit nombre de travaux récents (Karr, Little et Mitchell, I966; Wright, Grainger et Marco, I966; ØRSKOv, FrASER et KAY, I969). Ces différents auteurs ont montré qu'une quantité importante d'amidon pouvait échapper aux fermentations du rumen et être digérée en majeure partie dans l'intestin grêle. Cependant, aucune expérience n'a été effectuée pour étudier la digestion de l'amidon du maiss simultanément dans le rumen et dans l'intestin. C'est l'objet de ce travail qui fait partie de l'étude que nous avons entreprise pour comparer la digestion de l'amidon des principales céréales qui sont utilisées dans l'alimentation du ruminant (Thivend et Journet, 1968).

Deux bœufs fistulisés ont été utilisés. L'un $(470 \mathrm{~kg})$ était porteur d'une canule du rumen et d'une canule du duodénum ; l'autre $(580 \mathrm{~kg})$ était porteur d'une canule du duodénum. Nous avons fait varier le rapport foin/aliment concentré de la ration dans les proportions suivantes : IoO-O, puis $75-25,50-50,25-75$ et o-100, sans changer la quantité de matière sèche ingérée qui correspondait à celle du foin offert lors de la première période. La quantité d'amidon ingéré a ainsi varié de I ooo à 3 ooo g/jour environ. L'aliment concentré (19,3 p. Ioo de matières azotées, 57,2 p. Ioo d'amidon dans la matière sèche) contenait $77,8 \mathrm{p}$. Ioo de maïs, Io,4 p. Ioo de tourteau d'arachide et Io,4 p. Ioo de tourteau de soja ; il était présenté sous forme broyée et agglomérée. Le fourrage était un foin de luzerne ( $19,4 \mathrm{p}$. Ioo de matières azotées dans la matière sèche) sous forme normale.

Les aliments ont été distribués simultanément, en deux repas égaux, à $5 \mathrm{~h}$ 30 et à $\mathbf{I} 7 \mathrm{~h} 30$. Les animaux ont reçu les cinq régimes au cours de cinq périodes successives comprenant chacune deux semaines d'adaptation et deux semaines de mesure. Nous avons étudié pour chaque régime, la digestibilité de l'amidon et des autres constituants de la ration, les produits terminaux de la digestion dans le rumen (acides gras volatils, acide lactique), les activités amylolytiques in vitro et cellulolytiques in vivo du jus de rumen. Sur chaque animal, nous avons effectué une fois par période, et entre deux repas consécutifs, pendant $\mathrm{I} 2$ heures, des prélèvements discontinus $(3 \mathrm{mn}$ toutes les I5 mn) par l'intermédiaire de la canule du duodénum. A partir de ces prélèvements, nous avons préparé des échantillons (un par heure) sur lesquels nous avons dosé l'amidon (THIvend, Mercier, Guilbot, 1965).

La quantité journalière de matière sèche ingérée a été sensiblement la même pendant les quatre premières périodes. En revanche, elle a diminué d'environ $40 \mathrm{p}$. roo lorsque les animaux ont reçu uniquement l'aliment concentré, ce qui limite la signification de certains résultats (digestibilité, transit de l'amidon au niveau du duodénum) obtenus au cours de cette période. 
P. THIVEND, M. JOURNET

\begin{tabular}{|c|c|c|c|}
\hline \multirow{3}{*}{$\begin{array}{l}\dot{\vec{\theta}} \\
\dot{3} \\
\dot{0}\end{array}$} & 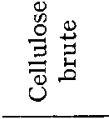 & 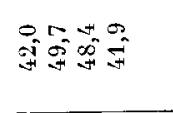 & \multirow{15}{*}{ 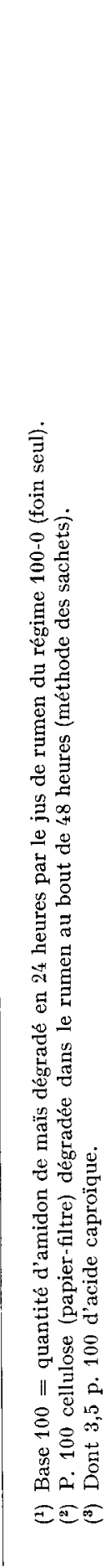 } \\
\hline & 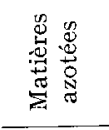 & 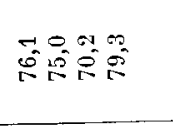 & \\
\hline & 惫惫 & 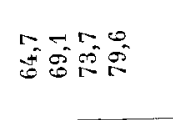 & \\
\hline \multirow{2}{*}{ 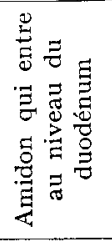 } & 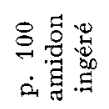 & 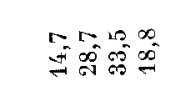 & \\
\hline & $\frac{10}{00}$ & 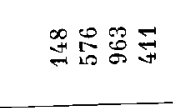 & \\
\hline 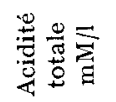 & & 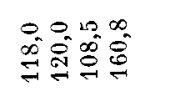 & \\
\hline 㺼 & $\begin{array}{l}\tilde{u} \\
\wedge\end{array}$ & 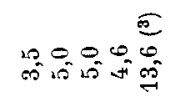 & \\
\hline 형 & $\vec{v}$ & 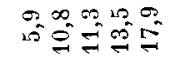 & \\
\hline 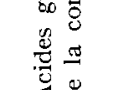 & $s$ & 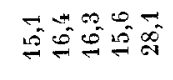 & \\
\hline $\begin{array}{l}\stackrel{\Xi}{\doteq} \\
\dot{\Leftrightarrow}\end{array}$ & $\omega$ & 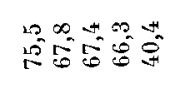 & \\
\hline 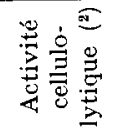 & & 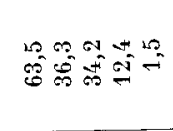 & \\
\hline 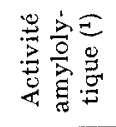 & & 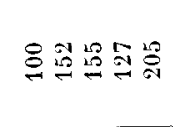 & \\
\hline 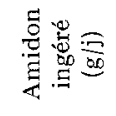 & & 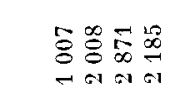 & \\
\hline 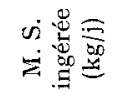 & & $\begin{array}{l}30=0 \\
30=0\end{array}$ & \\
\hline 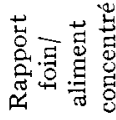 & & 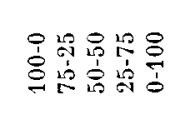 & \\
\hline
\end{tabular}


Le C. U. D. de la ration a augmenté avec le pourcentage d'aliment concentré. Il est passé de 64,7 à 79,6 pour la matière sèche, de 65,3 à $8 \mathbf{r}, 4$ pour la matière organique lorsque le rapport foin/aliment concentré variait de roo-o à $25-75$. Le C. U. D. de la cellulose brute Weende a légèrement augmenté tant que le pourcentage d'aliment concentré n'a pas dépassé $50 \mathrm{p}$. roo; il a diminué ensuite. Le C. U. D. des matières azotées a peu varié par suite de la constance de la teneur en azote de la ration au cours de l'expérience. L'amidon a toujours été entièrement digéré.

L'activité amylolytique et la composition du mélange d'acides gras volatils du jus de rumen ont été modifiées (tabl. I) lorsqu'une partie du foin (25 p. Ioo) a été remplacée par de l'aliment concentré, mais elles n'ont pas varié lorsque le rapport foin/aliment concentré est passé de 75-25 à 25-75. Seule, l'acidité totale du jus de rumen a augmenté pour le régime 25-75. L'activité cellulolytique a diminué au fur et à mesure que la quantité d'amidon ingéré augmentait. La production d'acide lactique a toujours été négligeable, y compris pendant la période au cours de laquelle les animaux n'ont reçu que de l'aliment concentré, bien que les autres produits terminaux et les activités microbiennes du rumen aient été très modifiés.

La quantité d'amidon entrant dans l'intestin a varié de $\mathbf{I}^{8} 8 \mathrm{~g}$ pour le régime $75^{-25}$ à $963 \mathrm{~g}$ pour le régime 25-75, ce qui représente un pourcentage important de l'amidon ingéré (de $\mathrm{I}_{4,7} \mathrm{à}$ 33,5 p. IOO). Nos résultats sont en accord avec les données de KARR, LitTle et Mitchell (1966) et confirment l'importance de la digestion intestinale de l'amidon du maïs cru. Lorsque les animaux n'ont reçu que l'aliment concentré ( $5^{\mathrm{e}}$ période), la part de l'amidon digéré en dehors du rumen a diminué (r 8,8 p. Ioo) probablement à cause des faibles quantités d'aliment concentré ingéré et d'une augmentation du temps de séjour de cet aliment dans le rumen.

La digestion de l'amidon de maïs chez le Ruminant est donc très différente de celle de l'amidon d'orge que nous avons étudiée précédemment (ThIVeno et Journet, 1968) ; elle peut se caractériser de la façon suivante :

- Les proportions d'acides gras volatils dans le rumen ne sont pas modifiées tant que la quantité de maïs dans la ration n'est pas supérieure à $60 \mathrm{p}$. Ioo. En revanche, les orientations fermentaires varient lorsque la ration contient plus de 45 p. Ioo d'orge.

- Une quantité importante d'amidon de maiss peut échapper aux fermentations du rumen et être transformée en glucose dans l'intestin alors que la quasi-totalité de l'amidon de l'orge est dégradée dans le rumen. En conséquence, le maïs, comparé à l'orge, doit favoriser l'engraissement des bovins et des ovins puisqu'il est digéré pour une part importante sous forme de glucose, lequel est mieux utilisé pour la lipogénèse que les acides gras volatils (ARMStrong et BLAXTER, I961).

Rę̧u pour publication en janvier 1970.

\section{SUMMARY}

\section{UTILIZATION OF MAIZE STARCH BY RUMINANTS}

Trials were done on two bullocks with cannulae in the rumen and duodenum. Amylolytic activity and volatile fatty acid composition of rumen fluid were not affected as long as the proportion of maize in the diet did not exceed $60 \mathrm{p}$. 10o. Cellulolytic activity diminished as the ratio of concentrate to roughage increased. No lactic acid was formed on any diet. The amount of starch which escaped fermentation in the rumen rise from $14^{8} \mathrm{~g}\left(\mathrm{I}_{4} .8 \mathrm{p}\right.$. I0o) to $963 \mathrm{~g}(33.5 \mathrm{P}$. 1 oo of the intake of starch) per day when the proportion of concentrate feed in the diet varied from 25 to $75 \mathrm{p}$. Ioo. Utilization of maize starch by ruminants was very different from that of barley starch. 


\section{RÉFÉRENCES BIBLIOGRAPHIQUES}

Armstrong D. G., Blaxter K. L., I96r. The utilization of the energy of carbohydrates by ruminant. 2th Symp. energy metab., Wageningen, p. I78.

Karr M. R., Little C. O., Mitchell G. E., ig66. Starch disappearance from different segments of the digestive tract of steers. J. Anim. Sci., 25, 652-654.

Thivend P., Mercier Ch., Guilbot A., ig65. Dosage de l'amidon dans les milieux complexes. Ann. Biol. anim. Bioch. Biophys., 5, 513-526.

Thivend P., Journet M., Ig68. Utilisation digestive de l'amidon de l'orge chez le Ruminant. Ann. Biol. anim. Bioch. Biophys., 8, 449-45I.

Ørskov E. R., Fraser C., KAY R.N.B., r969. Dietary factors affecting the digestion of starch in the rumen and small and large intestine of early weaned lambs. Br. J. Nutr., 23, 217-226.

Wright P. L., Grainger R. 13., Marco G. J., I966. Post ruminal degradation and absorption of carbohydrate by the mature ruminant. $J$. Nutr., 88, 24I-246. 